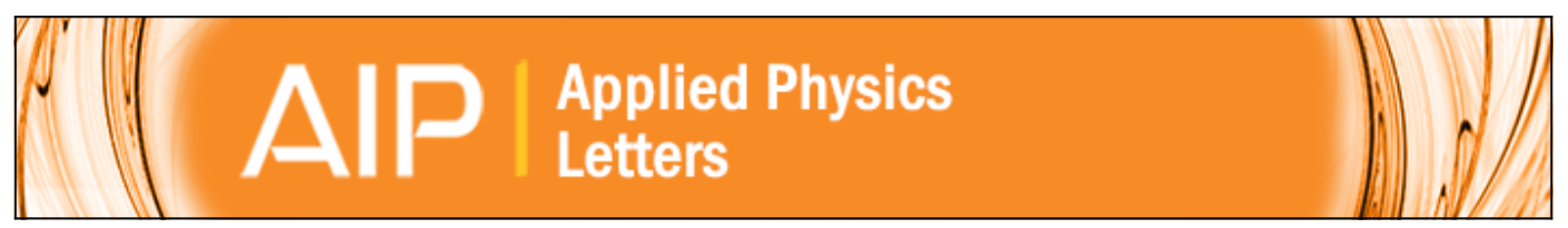

The effect of magnetic field on the intrinsic detection efficiency of superconducting single-photon detectors

J. J. Renema, R. J. Rengelink, I. Komen, Q. Wang, R. Gaudio, K. P. M. op 't Hoog, Z. Zhou, D. Sahin, A. Fiore,

P. Kes, J. Aarts, M. P. van Exter, M. J. A. de Dood, and E. F. C. Driessen

Citation: Applied Physics Letters 106, 092602 (2015); doi: 10.1063/1.4914182

View online: http://dx.doi.org/10.1063/1.4914182

View Table of Contents: http://scitation.aip.org/content/aip/journal/apl/106/9?ver=pdfcov

Published by the AIP Publishing

Articles you may be interested in

Inhomogeneous critical current in nanowire superconducting single-photon detectors

Appl. Phys. Lett. 105, 222602 (2014); 10.1063/1.4903071

Numerical analysis of detection-mechanism models of superconducting nanowire single-photon detector

J. Appl. Phys. 114, 214501 (2013); 10.1063/1.4836878

Intrinsic detection efficiency of superconducting nanowire single-photon detectors with different thicknesses

J. Appl. Phys. 108, 014507 (2010); 10.1063/1.3437043

Electrical characterization of superconducting single-photon detectors

J. Appl. Phys. 101, 054302 (2007); 10.1063/1.2709527

APL Photonics

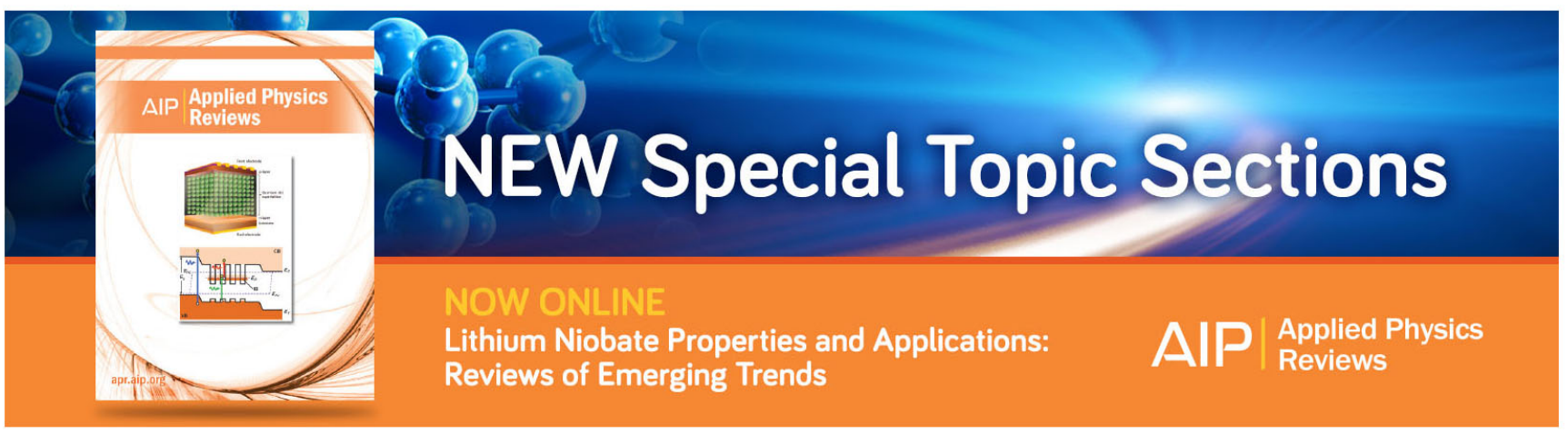




\title{
The effect of magnetic field on the intrinsic detection efficiency of superconducting single-photon detectors
}

\author{
J. J. Renema, ${ }^{1}$ R. J. Rengelink, ${ }^{1}$ I. Komen, ${ }^{1}$ Q. Wang,${ }^{1}$ R. Gaudio, ${ }^{2}$ K. P. M. op 't Hoog,${ }^{2}$ \\ Z. Zhou, ${ }^{2}$ D. Sahin, ${ }^{2,3}$ A. Fiore, ${ }^{2}$ P. Kes, ${ }^{1}$ J. Aarts, ${ }^{1}$ M. P. van Exter, ${ }^{1}$ M. J. A. de Dood, ${ }^{1}$ \\ and E. F. C. Driessen ${ }^{4,5}$ \\ ${ }^{1}$ Huygens-Kamerlingh Onnes Lab, Leiden University, Niels Bohrweg 2, 2333 CA Leiden, The Netherlands \\ ${ }^{2}$ COBRA Research Institute, Eindhoven University of Technology, P.O. Box 513, 5600 MB Eindhoven, \\ The Netherlands \\ ${ }^{3}$ Centre for Quantum Photonics, H. H. Wills Physics Laboratory, University of Bristol, Tyndall Avenue, \\ Bristol BS8 1TL, United Kingdom \\ ${ }^{4}$ Univ. Grenoble Alpes, INAC-SPSMS, 38000 Grenoble, France \\ ${ }^{5}$ CEA, INAC-SPSMS, 38000 Grenoble, France
}

(Received 27 October 2014; accepted 25 February 2015; published online 6 March 2015)

\begin{abstract}
We experimentally investigate the effect of a magnetic field on photon detection in superconducting single-photon detectors (SSPDs). At low fields, the effect of a magnetic field is through the direct modification of the quasiparticle density of states of the superconductor, and magnetic field and bias current are interchangeable, as is expected for homogeneous dirty-limit superconductors. At the field where a first vortex enters the detector, the effect of the magnetic field is reduced, up until the point where the critical current of the detector starts to be determined by flux flow. From this field on, increasing the magnetic field does not alter the detection of photons anymore, whereas it does still change the rate of dark counts. This result points at an intrinsic difference in dark and photon counts, and also shows that no enhancement of the intrinsic detection efficiency of a straight SSPD wire is achievable in a magnetic field. C 2015 AIP Publishing LLC.

[http://dx.doi.org/10.1063/1.4914182]
\end{abstract}

Nanowire superconducting single-photon detectors (SSPDs) ${ }^{1}$ are a crucial technology for single-photon detection in the infrared, since they can achieve detection efficiencies of up to $93 \%,{ }^{2}$ as well as low dark count rate, low jitter, and short reset time. ${ }^{3}$ These detectors consist of a narrow and thin wire of superconducting material, carrying a bias current.

While the broad outlines of the photodetection mechanism are known, there is as of yet no complete theory describing the response of such detectors. The present understanding of photodetection in SSPDs is as follows: ${ }^{4-12}$ when a photon is absorbed, a cloud of quasiparticles is created, which locally reduces the current-carrying capacity of the wire. Current is expelled from the absorption spot. If this diverted current is sufficiently strong, which depends on both the initial bias current and the energy of the photon, the Lorentz force may cause the unbinding of a vortex from the edge of the wire, leading to a measurable voltage pulse. Therefore, experiments on SSPDs in a magnetic field are a natural way of investigating the detection mechanism; one might even wonder whether the efficiency of the detector could be enhanced by applying a magnetic field.

In the present work, we study how an applied magnetic field directly affects the microscopic detection mechanism in a short section of wire. By using a single narrow active area in a bridge-like configuration, we avoid the question of current flow around curved sections of the device, which complicated the interpretation of previous experiments. ${ }^{13-15} \mathrm{We}$ find that it is the direct modification of the quasiparticle density of states in the superconductor that governs the magnetic field behaviour of SSPDs. In dirty-limit superconductors (such as thin-film $\mathrm{NbN}$ ), this density of states is modified by a bias current or a magnetic field. ${ }^{16}$ The effect of a magnetic field is therefore a homogeneous weakening of Cooper pairing, resulting in a higher detection efficiency at constant bias current. We identify three regimes. In the low-field regime (up to $\sim 50 \mathrm{mT}$ ), the current flow is sufficiently homogeneous. Bias current and magnetic field are completely interchangeable, as described by the Usadel equations. ${ }^{17}$ The relevant parameters of this theory do not depend on the illumination wavelength or on temperature in our measurement range, as is expected. In the intermediate regime $(50 \mathrm{mT}-200 \mathrm{mT})$, we still observe photon counts, but a higher current is required to achieve photodetection than predicted by the homogeneous theory. In the high-field regime $(200 \mathrm{mT})$, first photon and then dark counts are gradually extinguished when the field is increased. We attribute this to the presence of vortices in the wire.

We find that the enhancement of photon and dark counts on a single active spot obeys different field scales, pointing to a fundamental difference in the nature of the two. The field scale for the reduction of the critical current is smaller than the scale for the increase of the count rate. This leads us to conclude that no intrinsic enhancement of the detection efficiency of an SSPD under the influence of a magnetic field is possible.

Our experiments ${ }^{18}$ were performed on two different detectors: A $200 \mathrm{~nm}$ long bridge with a width of $150 \mathrm{~nm}$ (sample A), and a bowtie-shaped nanodetector ${ }^{19}$ with a width of $220 \mathrm{~nm}$ (sample B) and a thickness of $5 \mathrm{~nm} \mathrm{NbN}$ on a GaAs substrate. ${ }^{20}$ The samples were mounted in a Physical Properties Measurement System (PPMS) in a custom insert 
that allows optical coupling and high-frequency electronic readout. ${ }^{21}$ The orientation of the applied magnetic field was perpendicular to the film. In order to avoid hysteresis, all measurements were performed while increasing the magnetic field.

We illuminate our detectors with a continuous-wave laser with a wavelength of $826 \mathrm{~nm}$ and an optical power of $12 \mathrm{~mW}$. The illumination spot is approximately $2 \mathrm{~mm}$ in diameter. We have no control over the polarization, but it was kept constant during the experiment. We recorded the count rate during a $1 \mathrm{~s}$ interval at each current.

In Fig. 1, we plot a typical experimental result. The magnetic field was increased from $0 \mathrm{mT}$ to $300 \mathrm{mT}$ in steps of $30 \mathrm{mT}$. We observe an exponential increase of the count rate with bias current, followed by a saturation at higher currents, and a final exponential increase associated with dark counts, as is usually observed for this kind of detectors. ${ }^{22}$ The presence of a magnetic field shifts the curve towards lower currents. ${ }^{23} \mathrm{We}$ note that as the field is increased, a larger part of the count rate curve is dominated by dark counts. We conclude that photon counts and dark counts obey different field scales, even in our geometry where there is a single active area.

We have compared our results to the theory of Bulaevskii et al., ${ }^{10,11}$ which considers the effect of a magnetic field on the entry barrier of vortices. This theory predicts an exponential increase of count rate as a function of applied field, at constant bias current. As in the previous experiments, ${ }^{13,15}$ we find that the rate of exponential increase predicted by this theory is an order of magnitude away from the experimental value.

In Fig. 2, we plot those combinations of bias current and magnetic field which are required to achieve a constant count rate, from $1 / \mathrm{s}$ to $10^{5} / \mathrm{s}$. Since there is currently no theory which allows ab-initio calculation of the count rate, we therefore follow the usual experimental strategy $5^{5,6,24,25}$ of tracing the dependence of a point of constant count rate on one particular parameter. Our choice of working point is

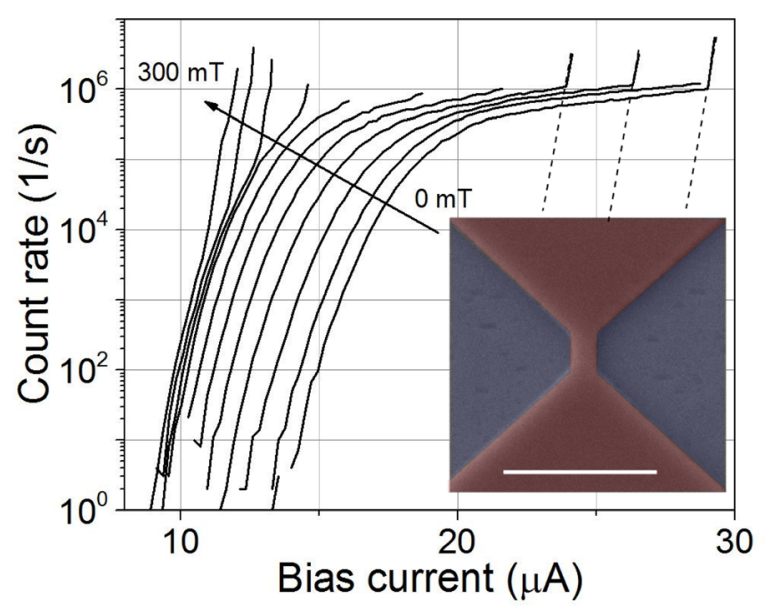

FIG. 1. Count rate of sample A, illuminated with $826 \mathrm{~nm}$ light at $\mathrm{T}=1.8 \mathrm{~K}$, for different magnetic fields ranging from $0 \mathrm{mT}$ to $300 \mathrm{mT}$, in steps of $30 \mathrm{mT}$. This measurement was not corrected for dark counts. The dashed lines are a guide to the eye indicating the part of the curve, where dark counts are dominant. Inset: false-colour SEM image of a detector ( $\mathrm{NbN}$ coloured red) nominally identical to sample A. The scale bar is $1 \mu \mathrm{m}$.

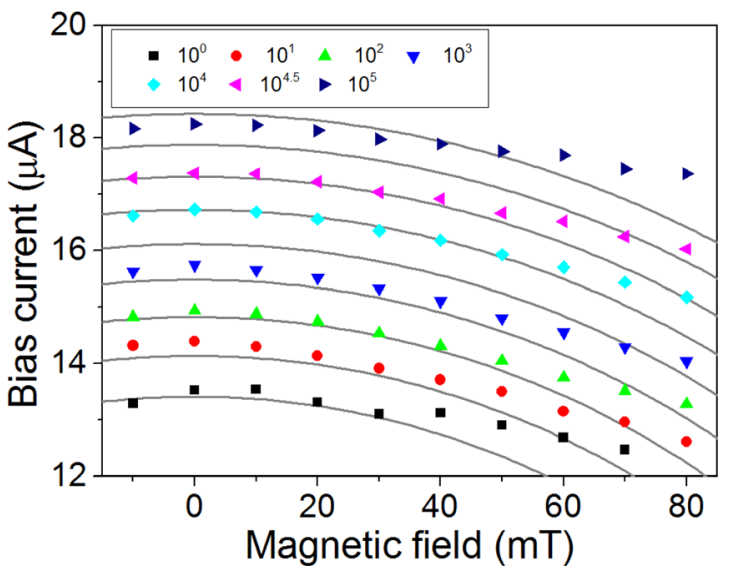

FIG. 2. Bias current at constant count rate as a function of magnetic field for sample A. The different colours and symbols correspond to different count rates, over five orders of magnitude. We find that for low magnetic fields (up to $\sim 50 \mathrm{mT}$ ) the required current to achieve a certain number of counts depends quadratically on applied magnetic field. The grey lines are equidistant ellipses calculated using the Usadel formalism.

motivated by the fact that in the steeply rising flank of the count rate the internal efficiency of the detector varies as it reaches the threshold current for that particular photon energy, ${ }^{5}$ whereas it is saturated at higher currents. ${ }^{26}$

For low magnetic fields $B \lessgtr 50 \mathrm{mT}$, the resulting contours of constant count rate lie on a series of concentric ellipses, which we have plotted in Fig. 2. For sample B, we similarly find concentric ellipses (not shown). In the measurement regime reported here, the dark count rate is negligible $(\ll 1 / \mathrm{s})$.

In Fig. 3, we turn to the temperature dependence of the magnetic-field response. We find that changing the temperature induces an overall shift in the contours of constant count rate, but that the shape of the ellipse is independent of temperature. We have also verified that this shape is independent of illumination wavelength by repeating the experiment with light of $405 \mathrm{~nm}$ and $1300 \mathrm{~nm}$ (not shown). The shift in count rate as a function of temperature at zero field is consistent with our previous results, ${ }^{6}$ where we showed that the temperature dependence of the SSPD

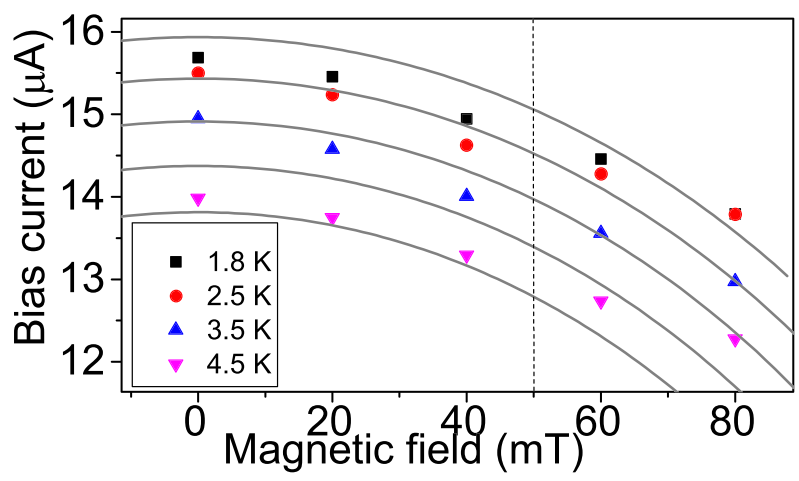

FIG. 3. Magnetic field dependence of the count rate at different temperatures for sample A. We plot the count rate required to obtain 1000 counts/s at different temperatures. The grey lines show the same equidistant ellipses as in Fig. 2. We find that changing the temperature induces only a shift in the current required to achieve a particular count rate but does not influence the magnetic field dependence. The dashed line indicates the limit of the applicability of our homogeneous theory. 
response is determined by the energy barrier for vortex entry.

We analyze these observations in terms of the microscopic theory for dirty-limit superconductivity, motivated by our analysis of the modification of the electronic state due to intrinsic pair breaking in similar films. ${ }^{27}$ For our film, $\lambda_{\perp} \gg w, d$, with $\lambda_{\perp}$ is the effective penetration length, and $w$ and $d$ are the width and thickness of the wire, respectively. Therefore, we assume a homogeneous current flow through our wire. In this case, the superconducting state is described by the homogeneous Usadel equation $^{17}$

$$
i E \sin \theta+\Delta \cos \theta-\Gamma \sin \theta \cos \theta=0
$$

where $E$ is the quasiparticle energy, $\theta$ is the pairing angle, $\Delta$ is the superconducting pairing potential, and $\Gamma$ is the pair breaking energy, representing a finite momentum of the Cooper pairs. A bias current $I_{b}$ and a perpendicular magnetic field have a similar effect in weakening the superconducting state, as was shown experimentally by Anthore et al. for one-dimensional aluminium wires. ${ }^{16}$ In this case, the depairing energy can be approximated by

$$
\begin{aligned}
& \frac{\Gamma}{\Delta}=\left(\frac{\Delta}{U(\Gamma)} \frac{I_{b}}{I_{\Gamma}}\right)^{2}+\left(\frac{B}{B_{\Gamma}}\right)^{2}, \\
& \frac{U(\Gamma)}{\Delta} \approx \frac{\pi}{2}-1.8 \frac{\Gamma}{\Delta}-\left(\frac{\Gamma}{\Delta}\right)^{2},
\end{aligned}
$$

where $I_{\Gamma}=\sqrt{2} \Delta / e R(\xi)$ and $B_{\Gamma}=\sqrt{6}(\hbar / e w \xi)$ are characteristic current and field scales for depairing, respectively, with $R(\xi)$ is the resistance of a section of the wire one coherence length $\xi$ long.

We note that the structure of these equations is compatible with our experimental observations at low fields: they define a series of concentric ellipses in the $I-B$ plane, connecting points with equal value of $\Gamma / \Delta$. For a more quantitative analysis of $I_{\Gamma}$ and $B_{\Gamma}$, we have determined the coherence length $\xi=3.9 \mathrm{~nm}$ from the slope of the upper critical field at the critical temperature. To evaluate $R(\xi)=7.2$ $\Omega$, we have assumed a homogeneous sheet resistance of our $\mathrm{NbN}$ film. We have determined the value of $\Delta=1.9 \mathrm{meV}$ at $\mathrm{T}=1.5 \mathrm{~K}$ using scanning tunnelling spectroscopy on a piece of the same film that was used to fabricate the detectors. In the STM tunneling spectra, we observe slightly rounded-off coherence peaks, consistent with the presence of an intrinsic pair breaker $\Gamma \approx 100 \mu \mathrm{eV}$, as was found previously on NbTiN and TiN films with similar resistivity. ${ }^{27,28}$ The presence of this pair breaker, which is larger than the pair breaking induced by applied current or field does not change the analysis that we present here, since it is a constant background to which the other pair breakers are added. Using these values, we estimate $I_{\Gamma}=180 \pm 20 \mu \mathrm{A}$ and $B_{\Gamma}=2.7 \mathrm{~T}$ for sample $\mathrm{A}$, and $I_{\Gamma}=330 \pm 20 \mu \mathrm{A}$ and $B_{\Gamma}=1.8 \mathrm{~T}$ for sample $B$. These values were used in generating the curves in Figures 2 and 3; the only remaining freedom is the dependence between the count rate $C$ and the normalized pair breaking energy $C(\Gamma / \Delta)$, which as discussed above is unknown.

From the excellent agreement between theory and experiment at magnetic field values $\mathrm{B} \lesssim 50 \mathrm{mT}$, we conclude that in this regime the count rate of the detector is determined only by a homogeneous weakening of the superconducting state, which can be described by the depairing energy $\Gamma$. This implies that the only way in which the magnetic field affects the detection mechanism is through the electronic state of the superconducting film before a photon is absorbed. This picture is re-enforced by the fact that the effects of magnetic field and temperature are independent: the field response is set by the film, whereas the temperature response is set by the barrier for a vortex entering the wire when a detection event occurs.

In Fig. 4, we plot the field dependence of a representative count rate (1000/s) and the field dependence of the critical current for a wider range of magnetic fields. We phenomenologically distinguish three regimes, independent of the chosen count rate. In the first regime, up to $\mathrm{B} \lesssim$ $50 \mathrm{mT}$, our data follow the prediction from the homogeneous theory. In the second regime $(50 \mathrm{mT}<B<200 \mathrm{mT})$, more current is required to produce detection events with a given probability than predicted by the homogeneous theory. In the third regime $(B>200 \mathrm{mT})$, the count rate is almost independent of the applied field. However, the critical current continues to decrease and eventually, there is a count-rate dependent point, where the observed count rate is entirely comprised of dark counts, indicated in Fig. 4 with an asterisk. For regimes I and II, the current required to observe substantial dark counts is too high to affect the analysis presented here. We observe throughout our measurement range that the dark counts shift with the critical current (see inset of Fig. 3). At a magnetic field of approximately $1 \mathrm{~T}$, no detection events are observed anymore in a $1 \mathrm{~s}$ interval.

To understand the physical meaning of the three regimes, we turn to the critical current measurements shown in the top panel of Figure 4. We observe linear decay of the

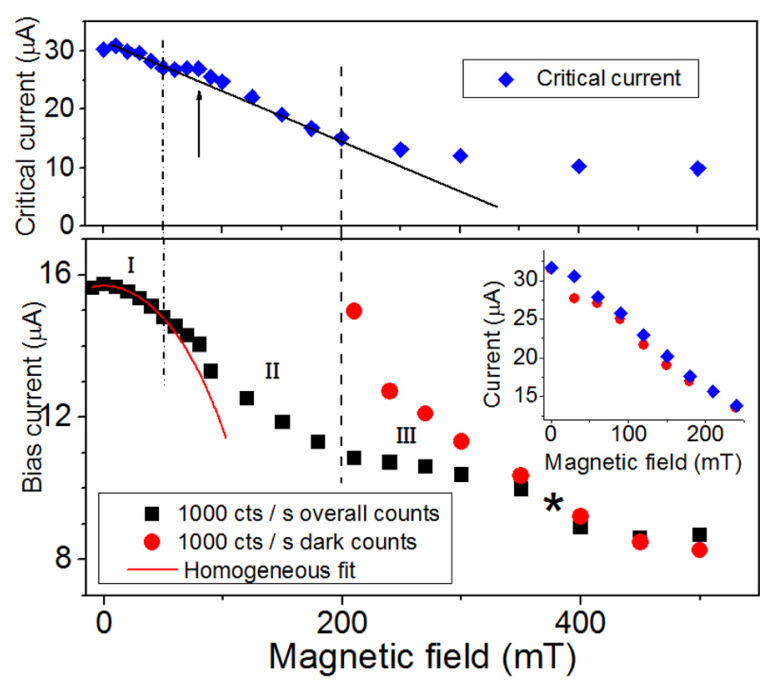

FIG. 4. Critical current (top) and curve of constant count rate (bottom) as a function of magnetic field for sample A. The black squares indicate count rate under illumination (photon counts + dark counts), the red circles indicate dark counts, and the blue diamonds indicate critical current. The asterisk marks the point where all observed counts are dark counts. The solid line in the top panel is a guide to the eye. The red curve is a plot of Eq. (2) for this count rate. We identify three regimes, demarcated by the two vertical lines. Inset: The low-field regime at high current. We plot the critical current and the current at which we observe a dark count rate of 1000/s. Note the difference in $y$-axis between the main figure and the inset. 
form $I_{c}(B)=I_{c}(0)\left(1-B / B_{0}\right)$, with $B_{0}=375 \mathrm{mT}$, up to the point $I_{c}(B)=0.5 I_{c}(0)$. At higher fields, we obtain a powerlaw behavior $I_{c} \propto B^{\alpha}$, with $\alpha \approx-0.4$. In this regime, we observe that there is no sharp transition to the normal state. We interpret these results in the context of the extensive literature on the field dependence of the critical current of superconducting strips, where the transition from linear to power-law behaviour is interpreted as the transition from a regime of critical current set by induced depairing to a regime, where the critical current is set by flux flow. ${ }^{29-32}$ The transition from induced depairing to flux flow corresponds to the transition of regime II to regime III in Fig. 4.

One additional feature is indicated by an arrow in the critical current measurements around $80 \mathrm{mT}$, where the critical current is enhanced relative to the linear dependence. We interpret this feature as a single vortex which is pinned in our material. ${ }^{13}$ All our measurements were done in a geometry that is intrinsically photodetecting, and a photodetection event entails a transition of the wire the normal state and Joule heating. Therefore, in-field cooling occurs each time there is a detection event. At $90 \mathrm{mT}$, we meet the criterion ${ }^{33}$ for entry of the first vortex $B \approx \Phi_{0} / w^{2}$. We conclude that while there is still an edge barrier at $\mathrm{B}=80 \mathrm{mT}$, so that vortices cannot enter, apparently the pinning is strong enough that a vortex which is already there is not expelled. We note that Il'in et al. ${ }^{34}$ have seen comparable enhancements of the critical current that were due to vortices, albeit in the fluxflow regime.

From this, we infer the following explanation of our results: In regime I, the current flow is sufficiently homogeneous so that the response can be explained by a homogeneous degradation of the superconducting state, described by the homogeneous Usadel equation. At the beginning of regime II, a vortex enters in the detector and is pinned in the material. This destroys the homogeneity. From the fact that the current which is required to obtain a detection event is higher than expected from theory, we infer that the presence of a vortex is detrimental to the detection process.

A full theory of regimes II and III is beyond the scope of the present work. It would have to take into account the direct effect of the magnetic field on the vortex barrier, the current distribution in our sample in the presence of vortices, and the associated local changes in $\Delta$. Any full microscopic theory of photodetection in SSPDs, even at zero magnetic field, would also need to take into account the intrinsic inhomogeneity that has been observed in similar films, ${ }^{35,36}$ and the observed intrinsic pair breaker, as it has been shown recently that these can give rise to an unexpected response to electromagnetic radiation. ${ }^{37}$

We have demonstrated that for low fields, the response of an SSPD to an applied magnetic field is set entirely by the effect that the field has on the electronic state of the material. In this regime, there is an interchange between bias current and applied magnetic field, in agreement with the homogeneous theory for dirty-limit superconductivity. Since the material parameters that enter this theory $\left(\lambda_{\perp}, \rho\right)$ are similar for all SSPDs found in literature, our results are not limited to $\mathrm{NbN}$ detectors. WSi, for example, has $\rho=200 \mu \Omega \mathrm{cm}$ and $\lambda=1400 \mathrm{~nm},{ }^{2,38}$ For the intermediate and high-field regimes, geometry and flux pinning properties become more relevant. Therefore, more diverse behaviour might be expected.

Our experiment disproves that the difference between photon and dark counts in a magnetic field is due to them originating from different points in the wire, as has been put forward by others. ${ }^{13,39,40}$ We conclude that there is a difference in the nature of photon and dark counts in SSPDs: photon counts scale with a field scale $B_{\Gamma}$ inherent to the material, whereas dark counts scale with the change in critical current under the influence of magnetic field, which depends on geometry. This difference between photon and dark counts is as of yet unexplained and carries implications for the design of SSPDs: it means that the only way in which an SSPD can be made more efficient by an applied magnetic field is by choosing a geometry where the critical current is not adversely affected by the applied field such as a spiral. ${ }^{41}$ For a straight wire, we conclude that no enhancement of the detection efficiency can be achieved by applying a magnetic field.

We would like to thank M. Hoek, M. Rosticher, R. Lusche, A. Engel, and C. Chapelier for discussions, J. Mesman for construction of the insert, M. Hesselberth, D. Boltje, K. Uhlirova, and I. M. Dildar for assistance with the experimental apparatus, B. Krama, R. Koehler, and A. van Amersfoort for assistance with the electronic circuit, and M. van Kralingen for his assistance in the early stages of this experiment. This work was part of the research programme of the Foundation for Fundamental Research on Matter (FOM), which is financially supported by the Netherlands Organisation for Scientific Research (NWO). It is also supported by NanoNextNL, a micro- and nanotechnology program of the Dutch Ministry of Economic Affairs, Agriculture and Innovation (EL\&I), and 130 partners, and by the Dutch Technology Foundation STW, Applied Science Division of NWO, the Technology Program of the Ministry of Economic Affairs under Project No. 10380. E.F.C.D. was financially supported by the CEA-Eurotalents programme.

${ }^{1}$ G. N. Goltsman, O. Okunev, G. Chulkova, A. Lipatov, A. Semenov, K. Smirnov, B. Voronov, A. Dzardanov, C. Williams, and R. Sobolewski, Appl. Phys. Lett. 79, 705 (2001).

${ }^{2}$ F. Marsili, V. B. Verma, J. A. Stern, S. Harrington, A. E. Lita, T. Gerrits, I. Vayshenker, and B. Baek, Nat. Photonics 7, 210 (2013).

${ }^{3}$ C. Natarajan, M. Tanner, and R. Hadfield, Supercond. Sci. Technol. 25, 063001 (2012).

${ }^{4}$ A. Engel and A. Schilling, J. Appl. Phys. 114, 214501 (2013).

${ }^{5}$ J. J. Renema, G. Frucci, Z. Zhou, F. Mattioli, A. Gaggero, R. Leoni, M. J. A. de Dood, A. Fiore, and M. P. van Exter, Phys. Rev. B 87, 174526 (2013).

${ }^{6}$ J. J. Renema, R. Gaudio, Q. Wang, Z. Zhou, A. Gaggero, F. Mattioli, R. Leoni, D. Sahin, M. J. A. de Dood, A. Fiore et al., Phys. Rev. Lett. 112, 117604 (2014).

${ }^{7}$ A. Zotova and D. Vodolazov, Supercond. Sci. Technol. 27, 125001 (2014).

${ }^{8}$ D. Y. Vodolazov, Phys. Rev. B 90, 054515 (2014).

${ }^{9}$ A. Eftekharian, H. Atikian, M. Akhlagi, A. Jafari Salim, and A. Hamed Majedi, Appl. Phys. Lett. 103, 242601 (2013).

${ }^{10}$ L. Bulaevskii, M. Graf, and V. Kogan, Phys. Rev. B 85, 014505 (2012).

${ }^{11}$ L. Bulaevskii, M. Graf, C. Batista, and V. Kogan, Phys. Rev. B 83, 144526 (2011).

${ }^{12}$ A. Gurevich and V. Vinokur, Phys. Rev. B 86, 026501 (2012).

${ }^{13}$ R. Lusche, A. Semenov, Y. Korneeva, A. Trifonof, A. Korneev, G. Goltsman, and H. Hübers, Phys. Rev. B 89, 104513 (2014). 
${ }^{14}$ R. Lusche, A. Semenov, K. Il'in, Y. Korneeva, A. Trifonov, A. Korneev, H.-W. Hübers, M. Siegel, and G. Goltsman, IEEE Trans. Appl. Supercond. 23, 2200205 (2013).

${ }^{15}$ A. Engel, A. Schilling, K. Il'in, and M. Siegel, Phys. Rev. B 86, 140506 (2012).

${ }^{16}$ A. Anthore, H. Pothier, and D. Esteve, Phys. Rev. Lett. 90, 127001 (2003).

${ }^{17}$ K. D. Usadel, Phys. Rev. Lett. 25, 507 (1970).

${ }^{18}$ See supplementary material at http://dx.doi.org/10.1063/1.4914182 for details of the experiment.

${ }^{19}$ D. Bitauld, F. Marsili, A. Gaggero, F. Mattioli, R. Leoni, S. Jahanmirinejad, F. Lévy, and A. Fiore, Nano Lett. 10, 2977 (2010).

${ }^{20}$ A. Gaggero, S. Jahanmirinejad, F. Marsili, F. Mattioli, R. Leoni, D. Bitauld, D. Sahin, G. J. Hamhuis, R. Nötzel, R. Sanjines et al., Appl. Phys. Lett. 97, 151108 (2010).

${ }^{21}$ We verified that the temperature of this custom insert was identical to the temperature measured on the block thermometer of the PPMS.

${ }^{22}$ C. M. Natarajan, A. Peruzzo, S. Miki, M. Sasaki, Z. Wang, B. Baek, S. Nam, R. H. Hadfield, and J. L. O’Brien, Appl. Phys. Lett. 96, 211101 (2010).

${ }^{23}$ From the fact that the curves have the typical shape for 1-photon detection and from the low count rate, we infer that multiphoton detection events do not play a role.

${ }^{24}$ R. Lusche, A. Semenov, K. Il'in, M. Siegel, Y. Korneeva, A. Trifonov, A. Korneev, G. Goltsman, D. Vodolazov, and H.-W. Hübers, J. Appl. Phys. 116, 043906 (2014).

${ }^{25}$ A. Engel, K. Inderbitzin, A. Schilling, R. Lusche, A. Semenov, D. Henrich, M. Hofherr, K. Il'in, and M. Siegel, IEEE Trans. Appl. Supercond. 23, 2300505 (2013), e-print arXiv:1210.5395v1.

${ }^{26}$ B. Baek, A. E. Lita, V. Verma, and S. W. Nam, Appl. Phys. Lett. 98, 251105 (2011).
${ }^{27}$ E. F. C. Driessen, P. C. J. J. Coumou, R. R. Tromp, P. J. de Visser, and T. M. Klapwijk, Phys. Rev. Lett. 109, 107003 (2012).

${ }^{28}$ P. C. J. J. Coumou, E. F. C. Driessen, J. Bueno, C. Chapelier, and T. M. Klapwijk, Phys. Rev. B 88, 180505 (2013).

${ }^{29}$ C. P. Bean and J. D. Livingston, Phys. Rev. Lett. 12, 14 (1964).

${ }^{30}$ B. L. T. Plourde, D. J. van Harlingen, D. Y. Vodolazov, R. Besselink, M. Hesselberth, and P. H. Kes, Phys. Rev. B 64, 014503 (2001).

${ }^{31}$ K. Il'in, D. Rall, M. Siegel, A. Engel, A. Schilling, A. Semenov, and H. Huebers, Physica C 470, 953 (2010).

${ }^{32}$ K. Il'in and M. Siegel, Physica C 503, 58 (2014).

${ }^{33}$ G. Stan, S. B. Field, and J. M. Martinis, Phys. Rev. Lett. 92, 097003 (2004).

${ }^{34}$ K. Il'in, D. Heinrich, Y. Luck, Y. Liang, and M. Siegel, Phys. Rev. B 89, 184511 (2014).

${ }^{35}$ B. Sacépé, C. Chapelier, T. I. Baturina, V. M. Vinokur, M. R. Baklanov, and M. Sanquer, Phys. Rev. Lett. 101, 157006 (2008).

${ }^{36}$ Y. Noat, V. Cherkez, C. Brun, T. Cren, C. Carbillet, F. Debontridder, K. Il'in, M. Siegel, A. Semenov, H.-W. Hübers et al., Phys. Rev. B 88, 014503 (2013).

${ }^{37}$ J. Bueno, P. C. J. J. Coumou, G. Zheng, P. J. de Visser, T. M. Klapwijk, E. F. C. Driessen, S. Doyle, and J. J. A. Baselmans, Appl. Phys. Lett. 105, 192601 (2014).

${ }^{38}$ A. Engel, J. Lonsky, X. Zhang, and A. Schilling, IEEE Trans. Appl. Supercond. 25, 2200407 (2015).

${ }^{39}$ G. Berdiyorov, M. Milosevic, and F. Peeters, Appl. Phys. Lett. 100, 262603 (2012).

${ }^{40}$ A. Zotova and D. Vodolazov, Supercond. Sci. Technol. 26, 075008 (2013).

${ }^{41}$ D. Henrich, L. Rehm, S. Dorner, M. Hofherr, K. Il'in, A. Semenov, and M. Siegel, IEEE Trans. Appl. Supercond. 23, 2200405 (2013). 